\title{
Consecuencias fisiopatológicas y anatómicas posgastrectomía vertical tubular como posibles causas de enfermedad por reflujo gastroesofágico
}

\author{
Italo Braghetto $\mathrm{M}$.
}

Departamento de Cirugí Hospital Clínico Universidad de Chile Santiago, Chile.

Recibido el 5 de marzo de 2018 , aceptado para publicación el 3 de mayo de

Correspondencia a: Dr Italo Braghetto M. ibraghet@hcuch.cl

\section{Physiopathological and anatomical consequences post tubular vertical gastrectomy as possible causes of gastro-esophageal reflux disease}

Tubular vertical gastrectomy (GVT) or sleeve gastrectomy has been validated as an effective bariatric procedure to treat patients with morbid obesity. Anatomical and pathophysiological changes have been described in the esophagogastric junction that may be related to the pathogenesis of gastroesophageal reflux disease. This article shows the results of our own experience and the literature regarding changes in esophageal and gastric function, the acid reflux test, endoscopic and radiological studies in patients undergoing GVT. It is concluded that GVT can present anatomical and physiopathological changes that are associated with the appearance of gastroesophageal reflux disease.

Key words: tubular vertical gastrectomy; gastroesophageal reflux.

\section{Resumen}

La gastrectomía vertical tubular (GVT) o en manga ha sido validada como un procedimiento bariátrico efectivo para tratar pacientes con obesidad mórbida. Se han descrito modificaciones anatómicas y fisiopatológicas en la unión esofagogástrica que pueden estar relacionados con patogénesis de la enfermedad por reflujo gastroesofágico. En este artículo se muestran los resultados de nuestra propia experiencia y de la literatura respecto de los cambios en la función esofágica y gástrica, la prueba de reflujo ácido, los estudios endoscópicos y radiológicos en pacientes sometidos a GVT. Se concluye que la GVT puede presentar cambios anatómicos y fisiopatológicos que se asocian a la aparición de enfermedad por reflujo gastroesofágico.

Palabras clave: gastrectomía vertical tubular; reflujo gastroesofágico.

\section{Introducción}

La gastrectomía vertical tubular (GVT) o en manga ha sido validada como un procedimiento bariátrico efectivo para tratar pacientes con obesidad mórbida, mostrando una pérdida media de exceso de peso entre $60 \%$ y $70 \%$ durante el primer año ${ }^{1-7}$. Se han descrito cambios manométricos y aumento significativo exposición esofágica al ácido debido a modificaciones anatómicas y fisiopatológicas en la unión esófago-gástrica que pueden estar relacionados con patogénesis de la enfermedad por reflujo gastroesofágico (ERGE) ${ }^{8-18}$. La mayoría de los estudios exploran a los pacientes usando solo endoscopia, sin embargo, el uso de la manometría, el tránsito esofagogástrico con sulfato de bario, o la prueba de reflujo ácido no se han realizado rutinariamente. Es muy conocido que una de las barreras más importantes que protegen el esófago del reflujo es el esfínter esofágico inferior (EEI). Esta estructura anatómica y funcional difiere de otros esfínteres en la disposición de las fibras musculares. El EEI no es un anillo, está compuesto por fibras musculares longitudinales, las "claps fibers" y fibras oblicuas situadas en el esófago distal y el cardias, que establecen un segmento de alta presión de $4 \mathrm{~cm}$ de esófago abdominal que evita el reflujo del contenido gástrico hacia el esófago $^{10}$. Esta estructura anatómica se modifica cuando se realiza una GVT o manga, comprometiendo la eficiencia de la EEI, lo que provoca una disminución 
en la presión de reposo de este esfínter (Figura 1). Una gastrectomía de manga ancha probablemente no produce estos cambios anatómicos pero no es una técnica adecuada.

El objetivo de este artículo es mostrar nuestra propia experiencia y de la literatura respecto de los cambios en la función esofágica y gástrica, la prueba de reflujo ácido, los estudios endoscópicos y radiológicos en pacientes sometidos a GVT.

\section{Estudios manométricos y monitoreo de $\mathrm{pH}$ ácido intraesofágico}

Nuestro grupo publicó en 2010 los resultados del estudio de los cambios manométricos en EEI después de la gastrectomía vertical tubular, la presión de reposo EEI preoperatoria disminuyó significativamente y la longitud de la zona de alta presión también se vio afectada después de la cirugía ${ }^{10}$. Se reconoce que hasta ahora no hay consenso porque los estudios han mostrado resultados paradójicos ${ }^{8-19}$. Algunos documentos muestran una mayor presión de EEI después de la cirugía, Petersen y Kleidi ${ }^{15,16}$ informaron que la GVT aumentó significativamente la presión de reposo de EEI independientemente de la pérdida de peso y sugieren que esta cirugía puede proteger a los pacientes obesos de ERGE. Ellos piensan que esta diferencia se debe a problemas técnicos, explicando que la posición de la engrapadora en relación con el ángulo de His es un factor importante. Sin embargo, en los mismos artículos describieron una mayor puntuación de los síntomas y los estudios endoscópicos demostraron insuficiencia cardial, esofagitis y presencia de hernia hiatal en la mayoría de los pacientes. Es difícil encontrar una explicación para estos hallazgos. Rebecchi et $\mathrm{al}^{17}$, no encontraron cambios en EEI y Del Genio et $\mathrm{al}^{8}$, con la manometría de alta resolución mostró que la función del EEI no sufría cambios, pero mostró peristalsis ineficaz y mal vaciamiento esofágico, pero sorprendentemente, estos mismos autores informaron incompetencia cardial o hernia hiatal asociada con la presencia de síntomas de reflujo con índice de síntomas aumentado, esofagitis endoscópica y ERGE di novo en el 5,4\% de los pacientes. Por el contrario, otros autores ${ }^{14,18-22}$, han demostrado un efecto nocivo sobre la presión del EEI, lo que indica una disminución de la presión del LES. Burgerhart ${ }^{14}$ observó una disminución en la presión de reposo LES de 18,3 \pm 9,2 a 11,0 \pm 7,0 $\mathrm{mmHg}(\mathrm{p}=0,02)$ medida por manometría de alta resolución. Gorodner, Sioka y Treidl encontraron una disminución en la longitud total y la presión de reposo LES posoperatoria en el $72 \%$ de los pacientes ${ }^{18-20}$. Valezi ${ }^{21}$, recientemente, confirma que casi la mitad de los pacientes tienen EEI hipotónico post GVT, cuya presión de reposo preoperatoria cayó de $26,5 \pm 9,1 \mathrm{mmHg}$ a $12,6 \pm 8,7 \mathrm{mmHg}$ en el posoperatorio $(\mathrm{p}=0,0001)$ (Tabla 1$)$.

En realidad, la manometría de alta resolución puede proporcionar información precisa sobre la morfología del EEI después de la manga y demostrar que la separación creciente entre EEI y crura diafragmática puede causar un aumento gradual y significativo en el reflujo ${ }^{22-25}$.

\section{Prueba de reflujo ácido después de GVT}

Las modificaciones en el EEI pueden estar asociadas con un aumento del reflujo de ácido en el esófago. En este sentido, se han publicado pocos estudios que informaron datos después de la monitorización del $\mathrm{pH}$ de $24 \mathrm{~h}^{14,18,25-29}$ (Tabla 2). Burgerhart $^{14}$ observó un aumento significativo en la exposición esofágica al ácido medida por $\mathrm{pH} /$ impedanciometría de 24 h de 4,9+3,5 a $12 \pm 10,4$ $\mathrm{mmHg}(\mathrm{p}=0,02)$ y puntaje de DeMeester de 12,6 $+10,1$ a $28,4+19$ con una diferencia de $193 \%$.

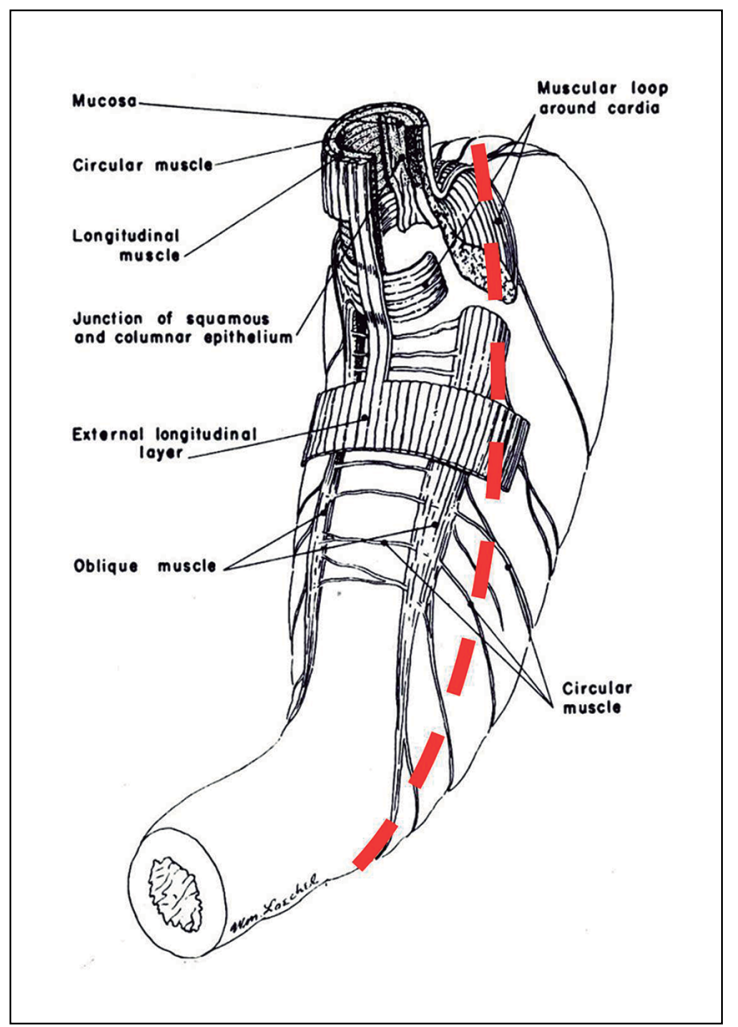

Figura 1. Línea de sección de fibras componentes del esfínter esofágico inferior durante la gastrectomía vertical tubular (Braghetto, Korn. IFSO, Montreal 2014). 
Tabla 1. Estudios manométricos posgastrectomía vertical tubular. Experiencia personal y datos de la literatura. Competencia esfínter esofágico inferior

\begin{tabular}{|c|c|c|c|c|c|}
\hline Autor (año) & $\mathbf{n}$ & Follow-up & $\begin{array}{l}\text { Presión } \\
\text { (mmHg) }\end{array}$ & $\begin{array}{c}\text { Largo } \\
(\mathrm{cm})\end{array}$ & Consecuencia clínica \\
\hline \multicolumn{6}{|c|}{ Disminuida ( $\mathrm{n}=5$ autores) } \\
\hline Braghetto (2010) & 23 & 6 meses & $10,5 \pm 6,1$ & $3,5 \pm 0,5$ & Síntomas de reflujo \\
\hline Braghetto (2018) & 35 & 5-9 años & $\begin{array}{c}15,3 \pm 4,2 \\
8,5 \pm 3,7\end{array}$ & $\begin{array}{l}4,0 \pm 0,2 \\
3,7 \pm 0,5\end{array}$ & $\begin{array}{l}\text { Sin síntomas }(n=6) \\
\text { Con esofagitis }(n=29)\end{array}$ \\
\hline Burgerhart (2014) & 20 & 3 meses & $11 \pm 0,7$ & $2,8 \pm 0,7$ & $62,8 \%$ Síntomas \\
\hline Gorodner (2015) & 14 & 12 meses & $12,4 \pm 4,5$ & $3,2 \pm 1,3$ & $64 \% \quad$ Esofagitis \\
\hline Sioka (2017) & 18 & $6-15$ meses & $10 \pm 8$ & $3,0 \pm 0$ & $32,2 \%$ Esofagitis \\
\hline Valezi (2018) & 73 & 12 meses & $12,6 \pm 8,7$ & - & No mencionado \\
\hline \multicolumn{6}{|c|}{ Aumento ( $n=2$ autores) } \\
\hline Petersen (2012) & 37 & 1 mes & $11 \pm 2,4$ & $3,2 \pm 0,5$ & $\begin{array}{l}\text { Hernia hiatal } \\
\text { Esofagitis }\end{array}$ \\
\hline Kleidi (2013) & 33 & 1.5 meses & $18 \pm 21,4$ & $3,8 \pm 0,8$ & Aumento de síntomas \\
\hline \multicolumn{6}{|c|}{ Sin cambios ( $n=2$ autores $)$} \\
\hline Rebecchi (2014) & 65 & 24 meses & $16,4 \pm 4,5$ & $4,1 \pm 0,3$ & $\begin{array}{l}\text { Reflujo de novo } \\
\text { Aumento de síntomas }\end{array}$ \\
\hline Del Genio (2014) & 25 & 13 meses & 22,4 & $3,8 \pm 0,3$ & No mencionado \\
\hline
\end{tabular}

Tabla 2. Estudios de pH esofágico gastrectomía vertical tubular

\begin{tabular}{|llccc|}
\hline Autor & (año) & \% tiempo $<\mathbf{4}$ & DeMesster's score & \% reflujo positivo \\
\hline Burgerhart & $(2014)$ & $12 \pm 10,4$ & - & - \\
Gorodner & $(2014)$ & $7,7 \pm 5,4$ & $24,8 \pm 19$ & $57 \%$ \\
\hline Del genio & $(2014)$ & $3,25($ rango $2-5)$ & $18,2($ rango $8-30,5)$ & - \\
Rebecchi & $(2014)$ & $3,5 \pm 1,4$ & $10,6 \pm 2,3$ & $10,8 \%$ \\
Thereaux & $(2016)$ & $5,6($ rango $2,5-9,5)$ & $23,8($ rango $10,1-39,5)$ & $68 \%$ \\
Doulami & $(2017)$ & 13,7 & $47 \pm 12,8$ & - \\
Ece & $(2017)$ & $21,8 \pm 15,6$ & $9,6 \pm 5,7 *$ & - \\
Georgia & $(2017)$ & - & - & $83,3 \%$ \\
Braghetto & $(2018)$ & $12 \pm 5,9$ & $43,5 \pm 22,5$ & $74,2 \%$ \\
\hline
\end{tabular}

*Cierre de pilares concomitante.

Gorodner ${ }^{18}$, encontró un aumento en la exposición ácida total de $3,8+3,1$ a 7,7 +5,4 después de la operación y la puntuación de DeMeester aumentó de 12,6 a $24,8(\mathrm{p}<0,05)(125 \%$ de incremento $)$. En el $36 \%$ de los pacientes se detectó reflujo "de novo" y el $21 \%$ presentó reflujo al puntaje patológico de DeMeester. En el estudio de Del Genio ${ }^{8}$, con pH/ impedanciometría de 24 h mostró un aumento del
$60 \%$ tanto de la exposición ácida del esófago como del número de eventos de reflujo no ácido en los períodos postprandiales al año de seguimiento. La explicación de estos hallazgos podría ser que las relajaciones transitorias inducen un aumento en los episodios de reflujo concomitante al aumento de los episodios de presión intragástrica, (fenómeno "squeeze"), que favorece el reflujo ácido positivo 
en el monitoreo del $\mathrm{pH}$ de $24 \mathrm{~h}$. Doulami ${ }^{26}$ publicó la puntuación de DeMeester de $47+12,78$ después de la cirugía casi 2,5 veces más que la puntuación preoperatoria y el porcentaje de tiempo total con $\mathrm{pH}<4$ también aumentó significativamente de 3,8 a $13,7 \%$. Thereaux ${ }^{27}$ encontró que el tiempo total a $\mathrm{pH}>4$ fue significativamente más alto después de la cirugía (aumenta de 1,6\% (0,7-2,9) a 5,6\% (2,5 a $9,5)$ y el puntaje DeMeester de 8,9 $(5,5-10,4)$ a 23,8 $(10,1-39,5)$. Ece $^{29}$ propuso realizar reparación concomitante de $\mathrm{HH}$ para disminuir el reflujo después de GVT. La evaluación posoperatoria con monitoreo de $\mathrm{pH}$ de $24 \mathrm{~h}$ demostró un tiempo total de $\mathrm{pH}<4$ y la mejora de la puntuación de DeMeester después cirugía. Al contrario, otros resultados ponen en duda el valor de la reparación concomitante de una hernia hiatal para prevenir el reflujo, pues en pacientes con reflujo ácido positivo preoperatoriamente no se encontraron cambios significativos poscirugía y además, se observó reflujo "de novo" en el $68 \%$ de los pacientes. Los estudios evaluados con gammagrafía con radionúclidos revelaron un aumento significativo de la ERGE del 6,25\% al 78,1\% en el período posoperatorio ( $\mathrm{p}<0,001)$, al mismo tiempo, la endoscopia digestiva alta mostró un aumento en la incidencia de esofagitis del $18,8 \%$ al $25 \%{ }^{30}$. En una publicación anterior, también encontramos la presencia de aumento de RGE con evaluación gammagráfica en casi el $75 \%$ de los $\operatorname{casos}^{12}$.

\section{Modificación de la presión intragástrica después de la GVT: Relación con la presión de EEI y las presiones del gradiente esofagogástrica y reflujo}

Otro efecto interesante de la GVT es la modificación en la presión intragástrica. Yehoshua et $\mathrm{al}^{31}$ observaron que la presión intragástrica basal no cambia después de la gastrectomía en manga, pero aumentó significativamente después de la oclusión del estómago e instilación con solución salina, lo que implica una disminución importante en la distensibilidad gástrica. Este fenómeno puede producir un aumento en el gradiente de presión esofagogástrica después de las comidas, lo que favorece el reflujo del contenido gástrico. En este sentido, las modificaciones anatómicas después de la cirugía, con alto riesgo de alteración de la morfología del EEI y modificaciones en el cuerpo gástrico (presencia de estenosis o dilatación del fondo) tienen una enorme importancia para el desarrollo de cambios fisiopatológicos en la presión intragástrica y en la presión de reposo del EEI. Mion y Tolone ${ }^{22,23,25}$, demostraron un aumento de la presión intragástrica (77\%), episodios de reflujo $(52 \%)$, asociados significativamente con síntomas de reflujo gastroesofágico y motilidad esofágica ineficaz post GVT. La capacidad gástrica y los diámetros del tubo gástrico también fueron significativamente menores en los pacientes con episodios de reflujo a la impedanciometría $(\mathrm{p}<0,01)$. Esto debería explicar por qué Himpens observó la disminución de los síntomas de ERGE después de un aumento inicial descrito. Después de un año, el tubo gástrico permanece estrecho, pero después de 3 años el tubo gástrico se ensancha y se aumenta, reduciendo la presión del gradiente esofagogástrica y, por lo tanto, el reflujo gastroesofágico ${ }^{32}$. En desacuerdo con este concepto, hemos observado pacientes con síntomas de reflujo y esofagitis hasta 9 años después de la GVT. No hemos realizado la medición de la presión intragástrica, pero es posible suponer que en pacientes con GVT, la presión intragástrica elevada y la dilatación cardial o hernia hiatal, son condiciones que promueven un gradiente de presión esofagogástrico elevado y reflujo subsecuente.

\section{Modificaciones anatómicas evaluadas por endoscopia y estudios radiológicos: Dilatación de cardias y hernia de hiato asociada con presión del esfinter esofágico inferior}

Concomitante a la manometría y prueba de reflujo ácido después de la cirugía, se evaluaron varios cambios de la anatomía de la unión esofagogástrica tanto por endoscopia como por tránsito de bario o tomografía computarizada. En el estudio de $\mathrm{Genco}^{33}$ con esofago-gastro-duodenoscopía, se encontró una migración hacia proximal de la línea " $Z$ " y reflujo esofágico en el 73,6\% y el 74,5\% de los casos, respectivamente. Experiencias recientes encontraron una correlación entre la apariencia radiológica de la GVT y el desarrollo de síntomas de ERGE. Sin embargo, la precisión de este tipo de evaluación es cuestionable debido a la naturaleza bidimensional del bolo baritado en comparación con la tomografía computada. En cualquier caso, ambos tipos de evaluación revelaron una modificación anatómica del EEI y del cuerpo del estómago ${ }^{34-36}$. Tai ${ }^{34}$ sugirió que el aumento de los síntomas de reflujo gastroesofágico después de la GVT es secundario a la presencia significativamente mayor de hernia hiatal $(6,1$ frente a $27,3 \%$ ), asociada a un aumento significativo de la esofagitis erosiva $(16,7$ vs $66,7 \%)(\mathrm{p}<0,001$ para ambos). Definitivamente para este autor la aparición de esofagitis erosiva después de VGT se relaciona con la presencia de una hernia hiatal después de la operación. Rengo ${ }^{37}$ estudió a 23 pacientes después de GVT con tomografía computarizada, RxEED con bario y endoscopia para identificar la dilatación esofágica, el desarrollo de neofundus, migración 
CIRUGíA AL díA

Tabla 3. Endoscopía y radiología posgastrectomía vertical tubular. Experiencia personal y datos de literatura

\begin{tabular}{|lll|}
\hline & & Literatura (Referencias) \\
Endoscopía $(\mathbf{n}=\mathbf{2 0 9})$ & & \\
Cardias dilatado con esofagitis & $57(27,2 \%)$ & $6,3-66,7 \% \%^{33-38}$ \\
Tipo A & 41 & $(71,9 \%)$ \\
Tipo B & 11 & $(19,3 \%)$ \\
Tipo C & 5 & $(8,7 \%)$ \\
Esófago de Barrett & $10(4,8 \%)$ & $15-17,2^{3} \%^{33,37,38}$ \\
Cardias dilatado con esofagitis & $79(37,8 \%)$ & \\
Hernia Hiatal & $11(5,3 \%)$ & $27-74 \%{ }^{33,37,38}$ \\
Estenosis mesogástrica & $3(1,4 \%)$ & $0,7-3,5 \%{ }^{11-13}$ \\
Radiología (n=93) & & \\
Reflujo presente & $42(45,2 \%)$ & $67 \%{ }^{11,33,38}$ \\
Cardias dilatado & $40(43 \%)$ & $60 \%{ }^{11,36,37,38}$ \\
Hernia Hiatal & $11(11,8 \%)$ & $60,8 \% 0^{36,37,38}$ \\
Estenosis mesogástrica & $3(3,2 \%)^{*}$ & $0,7-3,5^{11-13}$ \\
\hline
\end{tabular}

*bujía 34 French.

Tabla 4. Vaciamiento gástrico antes y después de gastrectomía vertical tubular

\begin{tabular}{|lll|}
\hline \multicolumn{3}{|c|}{ Experiencia propia Literatura } \\
\hline Vaciamiento gástrico para líquidos & & \\
$\mathrm{T} 1 / 2(\mathrm{~min})$ & & \\
Preop & $34,9 \pm 24$ & \\
Postop & $13,6 \pm 12(\mathrm{p}<0,01)$ & 1 autor (acelerado) \\
Vaciamiento gástrico para sólidos & & \\
Preop & $78 \pm 15$ & 2 autores sin cambios \\
Postop & $38 \pm 18(\mathrm{p}<0,01)$ & 10 autores acelerado \\
& &
\end{tabular}

(Referencias: 39-45). con respecto a este punto están de acuerdo con las otras experiencias informadas (Tabla 3 ).

\section{Vaciamiento gástrico}

Existe acuerdo entre la mayoría de los autores que confirman que el vaciamiento gástrico es acelerado después de GVT. Melissas y nosotros mismos informamos un vaciamiento gástrico más rápido para sólidos y líquidos ${ }^{12,39-44}$ (Tabla 4). Por el contrario, Bernstine ${ }^{45}$ no confirmó los mismos hallazgos para una comida semisólida sugiriendo que el vaciamiento gástrico no se ve afectado después de la GVT con preservación del antro. La técnica quirúrgica empleada en esos pacientes difiere de nuestra técnica porque resecamos parcialmente el antro, comenzando a $2-3 \mathrm{~cm}$ desde el píloro. Probablemente estas diferencias podrían explicar los diferentes resultados. Otro mecanismo importante involucrado en el vaciamiento gástrico rápido después de la GVT es la relación volumen/presión sugerida por Yehoshua et $\mathrm{al}^{31}$. La presión intragástrica aumenta significativamente en pacientes sometidos a esta operación, alcanzando hasta $40 \mathrm{mmHg}$ (32$58 \mathrm{mmHg}$ ) en comparación con $19 \mathrm{mmHg}(11-26$ $\mathrm{mmHg}$ ) en el estómago intacto. Esta presión intragástrica más alta refleja su menor distensibilidad y es un factor que promueve el vaciado gástrico rápido cuando se realiza la resección del antro.

\section{Reflujo duodeno gástrico}

Este tema no ha sido estudiado antes. Hemos estudiado a 20 pacientes después de la GVT, utilizando el método cintigráfico con ácido di-isopropiliminodiacético-tecnecio-99m, para detectar la presencia o ausencia de bilis radioactiva en el área gástrica predeterminada, 7 de ellos $(35 \%)$ presentaron reflujo duodeno-gástrico positivo. Tres de 7 pacientes $(42,8 \%)$ con reflujo biliar positivo tenían síntomas de reflujo y esofagitis tipo B (2 de ellos con esófago de Barrett y cuatro tenían síntomas pero con endoscopia normal. Entre los pacientes con reflujo duodenogástrico negativo, 13 tenían síntomas de reflujo pero solo 8 de ellos tenían esofagitis ( 3 con tipo B y 5 con esofagitis tipo A) ninguno con E. de Barrett. Los pacientes con síntomas de reflujo negativos habían sido sometidos previamente a una colecistectomía o presentaron un retraso en el vaciamiento vesicular después de la estimulación de la contracción. Aún faltan estudios para aclarar sobre el papel del reflujo biliar en pacientes con síntomas de reflujo y esofagitis post GVT pero estos resultados preliminares muestran que puede estar presente 
Tabla 5. Reflujo Duodeno gástrico cintigráfico y su relación con esofagitis erosiva post GVT

\begin{tabular}{|c|c|c|c|c|c|c|c|c|c|c|}
\hline \multirow[t]{2}{*}{ Síntomas $(\mathrm{n}=21)$} & \multirow[b]{2}{*}{$\mathbf{n}$} & \multicolumn{5}{|c|}{ Esofagitis } & \multicolumn{2}{|c|}{ Reflujo biliar } & \multicolumn{2}{|c|}{ Vesícula biliar } \\
\hline & & No & $\mathbf{A}$ & B & $\mathbf{C}$ & Barrett & Negativo & Positivo & No & Sí \\
\hline Leves & 5 & 5 & - & - & - & - & $3(60 \%)$ & $2(40 \%)$ & 5 & - \\
\hline Total & 21 & 9 & 4 & 6 & 1 & 1 & $14(66,6 \%)$ & $7(33,3 \%)$ & 14 & 7 \\
\hline
\end{tabular}

*2 con vaciamiento vesicular menos del $20 \%$ podría afectar presencia o no de reflujo biliar.

y si hay condiciones para el reflujo gastroesofágico podría ser la explicación para la aparición de Barrett post GVT (Tabla 5).

\section{Conclusión}

Hasta ahora, parece ser que el EEI se ve afectado y promueve el reflujo en una alta proporción de pacientes. Todavía tenemos que aprender mucho sobre los efectos fisiopatológicos de los procedimientos bariátricos para comprender a cabalidad los factores anatómicos, y cambios fisiológicos, pero con la información disponible hasta ahora, este procedimiento aumenta la ERGE y uno de los factores más importantes es el deterioro en la dinámica del EEI.

\section{Responsabilidades éticas}

Protección de personas y animales. Los autores declaran que para esta investigación no se han realizado experimentos en seres humanos ni en animales.

Confidencialidad de los datos. Los autores declaran que en este artículo no aparecen datos de pacientes.

Derecho a la privacidad y consentimiento informado. Los autores declaran que en este artículo no aparecen datos de pacientes. Este estudio ha sido efectuado de acuerdo a las normas éticas de nuestro hospital y de acuerdo a la declaración de Helsinky.

Conflicto de Interés: sin conflictos de interés.

\section{Referencias}

1. Sammour T, Hill AG, Singh P, Ranasinghe A, Babor R, Rahman H. Laparoscopic sleeve gastrectomy as a single-stage bariatric procedure. Obes Surg. 2010;20:271-5.

2. Deitel M, Gagner M, Erickson AL. Crosby RD. Third International Summit: Current status of sleeve gastrectomy. Surg Obes Relat Dis. 2011;7:749-59.

3. Himpens J, Dapri G, Cadiere G. A prospective randomized study between laparoscopic gastric banding and laparoscopic isolated sleeve gastrectomy: results after 1 and 3 years. Obes Surg. 2006;16:1450-6.

4. Chiu S, Birch DW, Shi X, Sharma AM, Karmali S. Effect of sleeve gastrectomy on gastroesophageal reflux disease. Surg Obes Relat Dis. 2011;7:510-5.

5. Mahawar KK, Jennings N, Balupuri S, Small PK. Sleeve gastrectomy and gastrooesophageal reflux disease: a complex relationship. Obes Surg. 2013;23:987-91.
6. Gagner M, Deitel M, Kalberer TL, Erickson AL, Crosby RD. The Second International Consensus Summit for Sleeve Gastrectomy. Surg Obes Relat Dis. 2009;5:476-85.

7. Rosenthal RJ. International Sleeve Gastrectomy Expert Panel Consensus Statement: best practice guidelines based on experience of 12,000 cases. Surg Obes Relat Dis. 2012;8:8-19.

8. Del Genio G, Tolone S, Limongelli P, Brusciano L, D'Alessandro A, Docimo G, et al. Sleeve Gastrectomy and Development of "De Novo" Gastroesophageal Reflux. Obes Surg. 2014;24:71-77.

9. Korn O, Csendes A, Burdiles P. Anatomic dilatation of he cardia and competence of the lower esophageal sphincer : a clinical and experiemnet study. J. Gastrointestinal Surg. 2000;4:398-406.

10. Braghetto I, Lanzarini E, Korn O, Valladares H, Molina JC, Henríquez A. Manometric changes of the lower esophageal sphincter after sleeve gastrectomy in obese patients. Obes Surg. 2010;20:357-62.

11. Braghetto I, Csendes A, Korn O, Valladares H, González P, Henríquez A. Gastroesophageal reflux disease after sleeve gastrectomy. Surg Laparosc Endosc Percutan Tech. 2010;20:148-53.

12. Braghetto I, Davanzo C, Korn O, Csendes A, Valladares H, Herrera E, et al. Scintigraphic evaluation of gastric emptying in obese patients submitted to sleeve gastrectomy compared to normal subjects. Obes Surg. 2009;19:1515-21.

13. Vogel SB, Drane WE, Woodward ER. Clinical and radionuclide evaluation of bile diversion by Braun enteroenterostomy: prevention and treatment of alkaline reflux gastritis. An alternative to Roux-en-Y diversion. Ann Surg. 1994;219:458-65.

14. Burgerhart JS, Schotborgh CA, Schoon EJ, Smulders JF, van de Meeberg PC, Siersema PD et al. Effect of sleeve gastrectomy on gastroesophageal reflux. Obes Surg. 2014;24:1436-41. 
15. Petersen WV, Meile T, Küper MA, Zdichavsky M, Königsrainer A, Schneider JH. Functional importance of laparoscopic sleeve gastrectomy for lower esophageal sphincter in patients with morbid obesity. Obes Surg. 2012;22:360-6.

16. Kleidi E, Theodorou D, Albanopoulos K, Menenakos E, Karvelis MA, Papailiou $\mathrm{J}$, et al. The effect of laparoscopic sleeve gastrectomy on the antireflux mechanism: can it be minimized? Surg Endosc. 2013;27:4625-30.

17. Rebecchi F, Allaix ME, Giaccone C, Ugliono E, Scozzari G, Morino M. Gastroesophageal reflux disease and laparoscopic sleeve gastrectomy: a physiopathologic evaluation. Ann Surg. 2014;260:909-14.

18. Gorodner V, Buxhoeveden R, Clemente G, Solé L, Caro L, Grigaites A. Does laparoscopic sleeve gastrectomy have any influence on gastroesophageal reflux disease? Preliminary results. Surg Endosc. 2015;29:1760-8.

19. Sioka E, Tzovaras G, Tsiopoulos F, Papamargaritis D, Potamianos S, Chatzitheofilou C, et al. Esophageal motility after laparoscopic sleeve gastrectomy. Clin Exp Gastroenterol. 2017;10:187-94.

20. Treidl D, Nieber D, Ben David K. Operative treatments for reflux after bariatric surgery: Current and emerging management options. J Gastrointest Surg. 2017:21:577-85

21. Valezi AC, Herbella FA, Mali-Junior J, Menezes MA, Liberatti M, Sato EO. Preoperative manometry for the selection of obese people candidate to sleeve gastrectomy. ABCD Arq Bras Cir Dig. 2017;30:222-4.

22. Mion F, Tolone S, Garros A, Savarino E, Pelascini E, Robert M, et al. Highresolution Impedance Manometry after Sleeve Gastrectomy: Increased Intragastric Pressure and Reflux are Frequent Events. Obes Surg. 2016;26:2449-56.

23. Tolone S, Savarino E, de Bortoli N, Frazzoni M, Furnari M, d'Alessandro A, et al. Esophagogastric junction morphology assessment by high resolution manometry in obese patients candidate to bariatric surgery. Int J Surg. 2016;28 Suppl 1:S109-13.

24. Hayat JO, Wan A. The effects of sleeve gastectomy on gastro-esophageal reflux and gastro-esophageal motility. Expert Rev Gastroenterol Hepatol. 2014;8:44552.

25. Tolone S, Savarino E, Yates RB. The impact of bariatric surgery on esophageal function. Ann N Y Acad Sci. 2016;138: 98-103.

26. Doulami G, Triantafyllou S, Natoudi M. Albanopoulos K, Filis K, Leandros E, et al. 24-h Multichannel intraluminal Impedance pHmetry 1 year after laparoscopic sleeve gastrectomy: an objective assessment of gastroesophageal reflux dideease. Obes Surg. 2017;27:74953.

27. Thereaux J, Bersamina C, Bretault M, Dusaussoy H, Lamarque D, Bouillot JL, et al. $\mathrm{pH}$ monitoring of gastroesophageal reflux before and after laparoscopic sleeve gastrectomy. BJS 2016;103:399-406.

28. Tolone S, de Cassan C, de Bortoli N, Roman S, Galeazzi F, Salvador R, et al. Esophagogastric junction morphology is associated with a positive impedance$\mathrm{pH}$ monitoring in patients with GERD. Neurogastroenterol Motil. 2015;27:142331.

29. Ece I, Yilamza H, Acar F, Colak B, Yormaz S, Sahin M. A New Algorithm to Reduce the Incidence of Gastroesophageal Reflux Symptoms after Laparoscopic Sleeve Gastrectomy. Obes Surg. 2017;27:1460-5

30. Sharma A, Aggarwal S, Ahuja V, Bal C. Evaluation of gastroesophageal reflux before and after sleeve gastrectomy using symptom scoring, scintigraphy, and endoscopy. Surg Obes Relat Dis. 2014;10:600-5.

31. Yehoshua RT, Eidelman LA, Stein M, Fichman S, Mazor A, Chen J, et al. Laparoscopic sleeve gastrectomy-volume and pressure assessment. Obes Surg. 2008;18:1083-8.

32. Himpens J, Dobbeleir J, Peeters G. Long term results of laparoscopic sleeve gastrectomy for obesity. Ann Surg. 2010;252:319-24.

33. Genco A, Soricelli E, Casella G, Maselli R, Castagneto Gissey L, Di Lorenzo N, et al. Gastroesophageal reflux disease and Barrett's esophagus after laparoscopic sleeve gastrectomy: a possible, underestimated long-term complication. Surg Obes Relat Dis. 2017;13:568-74.

34. Tai CM, Huang CK, Lee YC, Chang
CY, Lee CT, Lin JT. Increase in gastroesophageal reflux disease symptoms and erosive esophagitis 1 year afterlaparoscopic sleeve gastrectomy among obese adults. Surg Endosc. 2013;27:1260-6.

35. Karez WK, Kuester CK, Marianovic G, Suesslin D, Kotter E, Thomusch O, et al. 3D-MSCT gastric pouch volumetry in bariatric surgery: preliminray clinical results. Obes Surg. 2009:19:508-16.

36. Baumann T, Grienberger J, Pache G. Three dimensional stomach analysis with computed tomography after laparoscopic sleeve gastrectomy: sleeve dilatation and thoracic migration. Surg. Endosc. 2011;25:2323-9.

37. Rengo M, Bellini D, Iorio O, De Cecco CN, Rizzello M, Cavallaro G, et al. Role of preoperative imaging with multidetector computed tomography in the management of patients with gastroesophagealreflux disease symptoms after laparoscopic sleeve gastrectomy. Obes Surg. 2013;23:1981-6.

38. Felsenreich DM, Kefurt R, Schermann M, Beckerhinn P, Kristo I, Krebs M, et al. Reflux, Sleeve Dilation, and Barrett>s esophagus after Laparoscopic Sleeve Gastrectomy: Long-Term Follow-Up. Obes Surg. 2017;27:3092-101.

39. Melissas J, Daskalakis M, Koukouraki S, Askoxylakis I, Metaxari M, Dimitriadis E, et al. Sleeve gastrectomy- a food "limiting" operation. Obes. Surg. 2008;18:1251-6.

40. Melissas J, Koukouraki S, Askoxylakis J, Stathaki M, Daskalakis M, Perisinakis K, et al. Sleeve Gastrectomy- A restrictive procedure? Obes. Surg. 2007:17:57-62.

41. Michalsky D, Dvorak P, Belacek J, Kasalicky M. Radical resection of the pyloric antrum and its effect on gastric emptying after sleevegastrectomy. Obes Surg. 2013;23:567-73.

42. Kandeel AA, Sarhan MD, Hegazy T, Mahmoud MM, Ali MH. Comparative assessment of gastric emptying in obese patients before and after laparoscopic sleeve gastrectomy using radionuclide scintigraphy. Nucl Med Commun. 2015;36:854-62.

43. Sista F, Abruzzese V, Clementi M, Carandina S, Cecilia M, Amicucci G. The effect of sleeve gastrectomy on GLP-1 secretion and gastric emptying: a 
prospective study. Surg Obes Relat Dis. 2017;13:7-14.

44. Shah S, Shah P, Todkar J, Gagner M, Sonar S, Solav S.Prospective controlled study of effect of laparoscopic sleeve gastrectomy on small bowel transit time and gastric emptying half-time in morbidly obese patients with type 2 diabetes mellitus. Surg Obes Relat Dis. 2010 4;6:152-7.

45. Bernstine H, Tzioni_Yehoshua R, Groshar D, Beglaibter N, Shikora S, Rosenthal

CIRUGía AL díA

RJ, et al. Gastric emptying is nor affected by sleeve Gastrectomy. Scintigraphic Evaluation of gastric emptying after Sleeve gastrectomy without removal of the gastric Antrum. Obes. Surg. 2009:19:293-8. 